\title{
Solid liquid extraction of Jatropha seeds by microwave pretreatment and ultrasound assisted methods
}

\begin{abstract}
Jatropha curcas has a variety of uses which are of great economic significance. Jatropha oil can be used as fuel alternative and for making biodiesel that is supposed to overcome the source limitation problem. In this study, conventional, ultrasound assisted and microwave pretreatment solid liquid extraction of Jatropha seed were studied in terms of amount and quality of the extracted oil. The free fatty acid content which is an important oil quality index was also investigated for the obtained oil. Both ultrasonication and microwave pretreatment of the seeds had a positive effect on amount of yield. However, by application of ultrasound, more oil could be extracted compared with that obtained by conventional and microwave pretreatment extraction methods. The maximum amount of oil which could be extracted by conventional, ultrasound assisted and microwave pretreatment methods were 47.33, 51.4 and $49.36 \%$, respectively. Regarding the quality, oil extracted by conventional, ultrasound assisted and micmicrowave pretreatment extraction methods did not show any significant difference in terms of Free Fatty Acids (FFA) content.
\end{abstract}

Keyword: Biodiesel; Jatropha; Microwave pretreatment extraction; Solid liquid extraction; Ultrasound extraction 